\title{
Consequences of clustering in parasite-host relations of the cereal aphid Sitobion avenae: a simulation study
}

\author{
R. Rabbinge, A. G. Kroon and H. P. J. M. Driessen (Department of Theoretical \\ Production Ecology, Agricultural University, P.O. Box 430, 6700 AK Wagening- \\ en, Netherlands)
}

Accepted: 5 June 1984

\begin{abstract}
Quantitative effects of the patchy distribution of the English green aphid Sitobion avenae on its relation with the parasitoid Aphidius rhopalosiphi are studied by using a simulation model. The model computations are compared with field data. Different hypotheses on the parasitoid-host relation are tested. Due to the functional response and aggregation of the parasitoids, clustering increases the effect of parasitoids on population development of $S$. avenae, but the parasitoid does not prevent population upsurge even when it shows clear aggregation to host clusters.

Key-words: Sitobion avenae, Aphidius rhopalosiphi, clustering, biological control, simulation
\end{abstract}

Introduction. Insects are often aggregated in clusters in their habitat. This clustered distribution may affect their relationship with parasitoids or predators (Hassell, 1978; Morrison \& Strong, 1980).

This paper presents results of a simulation study of the influence of host clustering on the population dynamics of the cereal aphid Sitobion avenae F. on winter wheat, and its parasitoid Aphidius rhopalosiphi De Stephanie-Perez.

The simulation model. A simulation model of the population development of $S$. avenae (Rabbinge et al., 1979) was extended, to include a description of the parasitoidhost interaction and the population development of $A$. rhopalosiphi. The sigmoidal functional response curve for $A$. rhopalosiphi (Shirota et al., 1983) was introduced into the model. The figures for age-dependent oviposition rates were introduced for different age classes of the parasitoid. Only the first three aphid instars can be parasitized, and superparasitism is neglected. Each parasitized aphid dies immediately after parasitization.

The distribution. Clustering of the aphids is best described with the negative binomial distribution (Rabbinge \& Mantel, 1981). The parameter that determines clustering, $k$, was calculated for field counts in the period 1974 to 1979 by using the maximum likelihood method. The regression line of these $k$ values on $x$ is used in the model to estimate $k$ from the mean aphid density $\bar{x}(k=0.3128+0.0724 \bar{x}, r=$ $0.8, n=98)$. 
Host plants are arranged in density classes of cereal aphids according to the chance distribution of the negative binomial. The mean host density in each class is used to calculate the parasitization rate. Various assumptions about the degree of aggregation of the parasitoids are considered.

The effect of clustering is evaluated by comparing three simulations:

A. Both host and parasitoid are distributed at random.

$B$. The host has a negative binomial distribution and the parasitoid is distributed at random.

C. The host has a negative binomial distribution, and the parasitoids are distributed in proportion to local host density.

Results. Simulated results were compared with measured densities of cereal aphids per tiller during the 1981 growing season in a winter wheat field at Wageningen.

Predicted population development of the aphids varies only slightly between the different model calculations (Fig. 1). Without clustering of the aphids the influence of the parasitoids on the number of aphids is negligible. The simulated total number of aphids lies almost entirely within the confidence levels of the field data (A).

Clustering of the aphids increases the effect of the parasitoids even without aggregation of the parasitoids on tillers with high aphid density (B). This may be due to the sigmoidal form of the functional response curve. At the prevailing mean aphid densities the parasitization rate is more than proportional to the numbers of aphids per tiller.

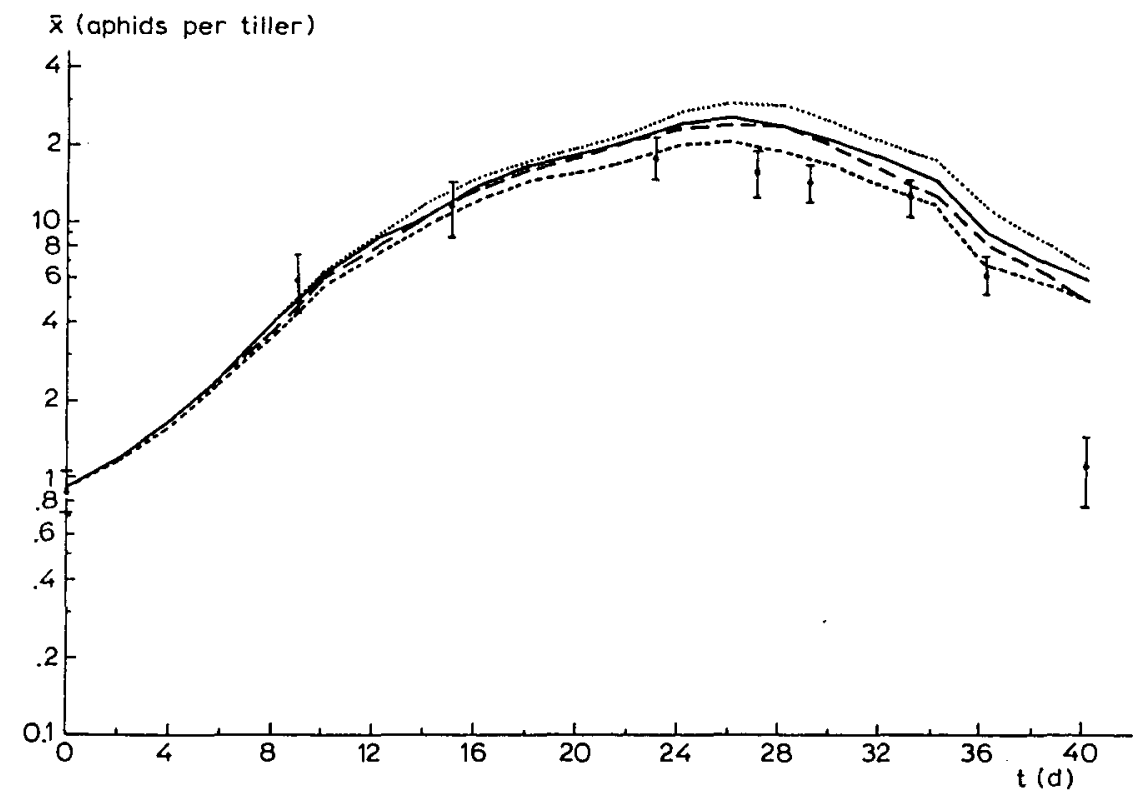

Fig. 1. Mean aphid density $(\tilde{x})$ in the field (-, with confidence interval), and the results of simulations $\mathrm{A}(-), \mathrm{B}(--)$ and $\mathrm{C}(-\ldots)$ (see text), and without parasitoids $\mathrm{D}(\ldots \ldots$.$) .$ 
Aggregation of the parasitoids in the clusters enhances this effect (C). Here, however, the aphid density is so much reduced that the parasitization rate per parasitoid is lower than in case B. At the end of the season this is not entirely compensated by the higher parasitoid density and the aggregation of the parasitoids.

Discussion. The simulated effect of the parasitoids is increased by introduction of clustering of the aphids and aggregation of the parasitoids. According to Waage \& Hassell (1982) this effect may be over-estimated by neglecting interference arising from encounters with other parasitoid adults, as well as hyperparasitism. In that case the effect of the parasitoids is even smaller. Little is known of the degree of aggregation of parasitoids in the field (Morrison \& Strong, 1980).

The functional response (Shirota et al., 1983) cannot be used for a proper estimate of the oviposition rates in the field, and the available data on percentage parasitism in the field are inaccurate, so a comparison with simulated results is of limited value.

Nevertheless, the results of the present simulations, and the consequences of the imperfections of the model, suggest that the effect of the parasitoids on aphid populations in the field is negligible.

\section{References}

Hassell, M. P.. 1978. The dynamics of arthropod predator-prey systems. Monographs in population biology no. 13, Princeton University Press, $237 \mathrm{pp}$.

Morrison, G. \& D. R. Strong Jr., 1980. Spatial variations in host density and the intensity of parasitism: Some empirical examples. Environmental Entomology 9: 149-152.

Rabbinge, R., G. W. Ankersmit \& G. A. Pak, 1979. Epidemiology and simulation of population development of Sitobion avenae in winter wheat. Netherlands Journal of Plant Pathology 85: 197-220.

Rabbinge, R. \& W. P. Mantel, 1981. Monitoring for cereal aphids in winter wheat. Netherlands Journal of Plant Pathology 87: 25-29.

Shirota, Y., N. Carter, R. Rabbinge \& G. W. Ankersmit, 1983. Biology of Aphidius rhopalosiphi, a parasitoid of cereal aphids. Entomologia Experimentalis et Applicata 34: 27-34.

Waage, J. R. \& M. P. Hassell, 1982. Parasitoids as biological control agents - a fundamental approach Parasitology 84: 241-268.

This synopsis is based on an M. Sc. thesis, Department of Theoretical Production Ecology, Agricultural University, Wageningen, 1983. Internal report of the Department of Theoretical Production Ecology. 131 pp., tables, figs., refs. Dutch.

Available as paper copy (order R016P, $f 30$ including postage) or microfiches (order R016M, $f$ 17,50 including postage) at: NARD, clo Pudoc, P.O. Box 4, 6700 $A A$ Wageningen, Netherlands (telex 45015 blhwg nl). 